\title{
Mammalian pre-mRNA branch site selection by U2 snRNP involves base pairing
}

\author{
Jian Wu and James L. Manley \\ Department of Biological Sciences, Columbia University, New York, New York 10027 USA
}

\begin{abstract}
SV40 early pre-mRNA is alternatively spliced to produce large $T$ and small $t$ mRNAs by use of different $5^{\prime}$ splice sites and a shared 3 '-splice site. The large $T$ splicing pathway uses multiple lariat branch sites, whereas small $t$ splicing, constrained by its small intron size, can use only one. We exploited this situation to test the hypothesis that RNA-RNA base pairing between U2 snRNA and the branch site sequence is important in mammalian pre-mRNA splicing by constructing and analyzing several mutations in the small $t$ pre-mRNA branch site (UUCUAAU). All of the mutations resulted in substantial decreases in small $t$ splicing relative to large $T$. To test whether these effects resulted from decreased base pairing with U2 snRNA, compensatory mutations were introduced at the appropriate positions (nucleotides 34-36) in a cloned human U2 gene. All branch site mutations tested (four separate single base substitutions and two triple mutations) were suppressed (i.e., small $t$ splicing was increased) by the appropriate $U 2$ mutations. These results establish that recognition of the poorly conserved mammalian pre-mRNA branch site sequence by U2 snRNP can involve base-pairing.
\end{abstract}

[Key Words: snRNP; base pairing; small t mRNA; large T mRNA]

Received June 16, 1989; accepted July 19, 1989.

Splicing of pre-mRNAs in eukaryotic cells is a complex process that requires several cis-acting sequences and a large number of trans-acting factors (for reviews, see Green 1986; Padgett et al. 1986; Maniatis and Reed 1987). Foremost among the latter are several small nuclear ribonuclear protein particles (snRNPs). It appears that splicing requires the participation of four separate snRNPs: U1, U2, U5, and U4/U6. U1 snRNP function involves recognition of the pre-mRNA $5^{\prime}$ splice site (Mount et al. 1983), and U2 snRNP function involves an interaction with the lariat branch site (Black et al. 1985). U5 snRNP may interact with the $3^{\prime}$ splice site (Chabot et al. 1985), whereas U4/U6 snRNP does not appear to contact the pre-RNA directly (Bindereif and Green 1987).

The interaction of Ul snRNP with the $5^{\prime}$ splice site involves base pairing between the $5^{\prime}$ end of U1 snRNA and the conserved $5^{\prime}$ splice site (CAG/GURAGU). This was suggested first from the observed base-pairing potential between these sequences (Lerner et al. 1980; Rogers and Wall 1980) and has been supported by a number of experimental observations. These include analysis of the effects of base substitutions in the premRNA consensus 5' splice site (Aebi et al. 1987; Weber and Aebi 1988) and the observation that binding of U1 snRNP to a $5^{\prime}$ splice site in vitro requires the $5^{\prime}$ end of U1 RNA (Chabot et al. 1985). The most conclusive evidence, however, comes from experiments showing that the inhibition of splicing brought about by a base substi- tution in a pre-mRNA $5^{\prime}$ splice site consensus (position +5 in the intron) can be suppressed in a transient expression assay by introducing a compensatory change at the appropriate position in a plasmid encoding U1 RNA (Zhuang and Weiner 1986). However, a mutation at the adjacent +3 position cannot be suppressed efficiently, suggesting that the role of the $5^{\prime}$ splice site consensus is not solely to base-pair with sequences in UI RNA. Suppressor mutations also have been used to show that base pairing plays a role in $5^{\prime}$ splice site recognition by Ul snRNP in yeast (Seraphin et al. 1988; Siliciano and Guthrie 1988).

The interaction between U2 snRNP and the premRNA branch site, at least in yeast, also involves basepairing. The sequence at the branch site (UACUAAC) is absolutely conserved, and mutations within it almost invariably reduce or inhibit splicing (e.g., Langford and Gallwitz 1983; Pikielny et al. 1983). As with U1 RNA, compensatory mutations in the yeast equivalent of $\mathrm{U} 2$ snRNA can suppress, in an allele-specific manner, two different base substitution mutations in the UACUAAC sequence (Parker et al. 1987). By analogy with selfsplicing group II introns (Schmelzer and Schweyen 1986), and also to optimize potential base pairing, it has been proposed that the adenosine functioning as the branch acceptor (underlined above) is bulged from the presumptive RNA-RNA helix (Parker et al. 1987).

The nature of the U2 snRNP-branch site interaction in metazoans is less clear. The branch site consensus se- 
quence (YNCURAY; Keller and Noon 1984; Ruskin et al. 1984; Zeitlin and Efstradiatis 1984) is only poorly conserved, and, in several cases, mutations in this consensus only have modest effects on splicing (e.g., Wieringa et al. 1984). Such mutations, however, often lead to the use of cryptic branch sites, which need bear little, if any, resemblance to the consensus sequence (Padgett et al. 1985; Ruskin et al. 1985). These results might be interpreted as arguing against base-pairing as an important determinant in branch site-U2 snRNP interactions. However, a potential base-pairing between the branch site and internal sequences in U2 snRNA, similar to that in yeast, has been noted (Parker et al. 1987). Also, analysis of the splicing of mutant $\beta$-globin pre-mRNAs containing duplicated $3^{\prime}$ splice site/branch site regions revealed that choice of $3^{\prime}$ splice site is determined by the branch site sharing the greatest homology with the consensus sequence (Reed and Maniatis 1988; Zhuang et al. 1989), indicating that the consensus is functionally important.

SV40 early pre-mRNA is alternatively spliced, by the use of two $5^{\prime}$ and a single $3^{\prime}$ splice site, to produce large $\mathrm{T}$ and small $\mathrm{t}$ mRNAs. Certain properties of the pathway by which this pre-mRNA is spliced suggested that it might be well-suited to test the hypothesis that recognition of the branch site in mammalian pre-mRNAs by U2 snRNP involves base-pairing. Large $\mathrm{T}$ splicing, which uses the promoter-proximal $5^{\prime}$ splice site, is somewhat unusual in that as many as 9 different nucleotides located between positions -18 and -32 , relative to the $3^{\prime}$ splice site, can function as branch acceptors (Noble et al. 1987,1988 ). This promiscuity appears to be due, at least in part, to the presence of multiple copies of the branch site consensus in this region (Noble et al. 1989). In contrast, small $t$ splicing uses principally one branch acceptor, the $A$ at position -18 . This is due to the very small size of this intron (66 nucleotides), which is at or near a minimum (Fu and Manley 1987; Fu et al. 1988a). Consistent with the notion that small $\mathrm{t}$ splicing is constrained to use only this branch site, a single-base substitution changing the $A$ at position -18 to $U$ reduces small $t$ splicing dramatically, relative to large $\mathrm{T}$ (Noble et al. 1988). These findings suggest that mutations at other positions in the small $t$ consensus might also reduce the relative efficiency of small $t$ splicing. Such mutations may then prove ideal for testing the hypothesis that branch site recognition by U2 snRNP involves base pairing, by allowing us to determine whether small $t$ splicing can be restored by compensatory mutations in U2 snRNA. The results of such experiments, which establish that base pairing does indeed occur, are presented below.

\section{Results}

Mutations in the small $t$ branch site consensus inhibit small $t$ splicing

Previously, we analyzed a series of mutants containing base substitutions that altered, singly or in combina- tions, the major branch acceptor nucleotides in the SV40 early pre-RNA (Noble et al. 1988). We observed that in transfected HeLa cells, large T splicing was not inhibited dramatically by any of these mutations, but small $t$ splicing was reduced significantly by $\mathrm{A} \rightarrow \mathrm{U}$ transversions at position -18 and, to a lesser extent, -19 . A double mutation encompassing both of these positions essentially eliminated small $t$ splicing.

To extend this analysis, we constructed several additional base substitutions in the branch site consensus surrounding the $\mathrm{A}$ at position -18 (see Fig. 1). The effects of these mutations then were determined by transfection into human 293 cells (Graham et al. 1977). These cells were used instead of HeLa for two reasons. First, the amount of small $t$ splicing, relative to large $T$, is significantly higher in 293 cells than in other cell types (Fu and Manley 1987), which facilitates analysis of the effects of mutations on small $t$ splicing. Second, because our ultimate aim was to determine the effects of compensatory mutations in U2 snRNAs expressed from cotransfected U2 genes, it was important to use cells in which the human U2 gene to be employed (Westin et al. 1984) is expressed efficiently. Primer extension analysis of RNA extracted from transfected 293 cells, using a 'marked' version of the U2 gene, revealed that significantly more U2 RNA accumulated in 293 cells than in HeLa cells (Y.M. Han et al., unpubl.), arguing again for the use of 293 cells in these experiments.

Nuclease S1 analysis of cytoplasmic RNA extracted from 293 cells transfected with the wild-type plasmid pSTER or with each of the mutants indicated in Figure 1, is shown in Figure 2. The ratio of small $t$ to large $T$ mRNA produced from pSTER in 293 cells was $\sim 1$. All of the mutations resulted in significant decreases in small $t$ splicing. Among the single-base substitutions, the most severe inhibition was caused by mutations that altered the conserved $U$ at position $-20 / 2$ bases upstream of the branch acceptor). This finding is similar to observations reported by Reed and Maniatis (1988) and Zhuang et al. (1989) and suggests that this $U$, which is situated at the center of the branch site consensus, plays a critical role in branch site selection. Small $t$ splicing was undetectable with the triple mutants SV-GGU and SV-AGU (results for SV-AGU not shown). The unspliced precursor RNA detected resulted from nuclear leakage, which we observed previously with 293 cells (Fu and Manley 1987).

\section{Branch site mutations can be suppressed by compensatory mutations in U2 snRNA}

To test whether RNA-RNA base pairing may play a role in the recognition of the branch site by U2 snRNA, we constructed a number of different mutations in the region of U2 snRNA hypothesized to base-pair with the branch site consensus (nucleotides +33 to +38 ; Parker et al. 1987). In each case, we not only introduced a mutation capable of restoring base-pairing but also an additional change that should not allow base pairing to occur (see Fig. 1). Derivatives of PSTER containing each of the 


\section{pSTER}
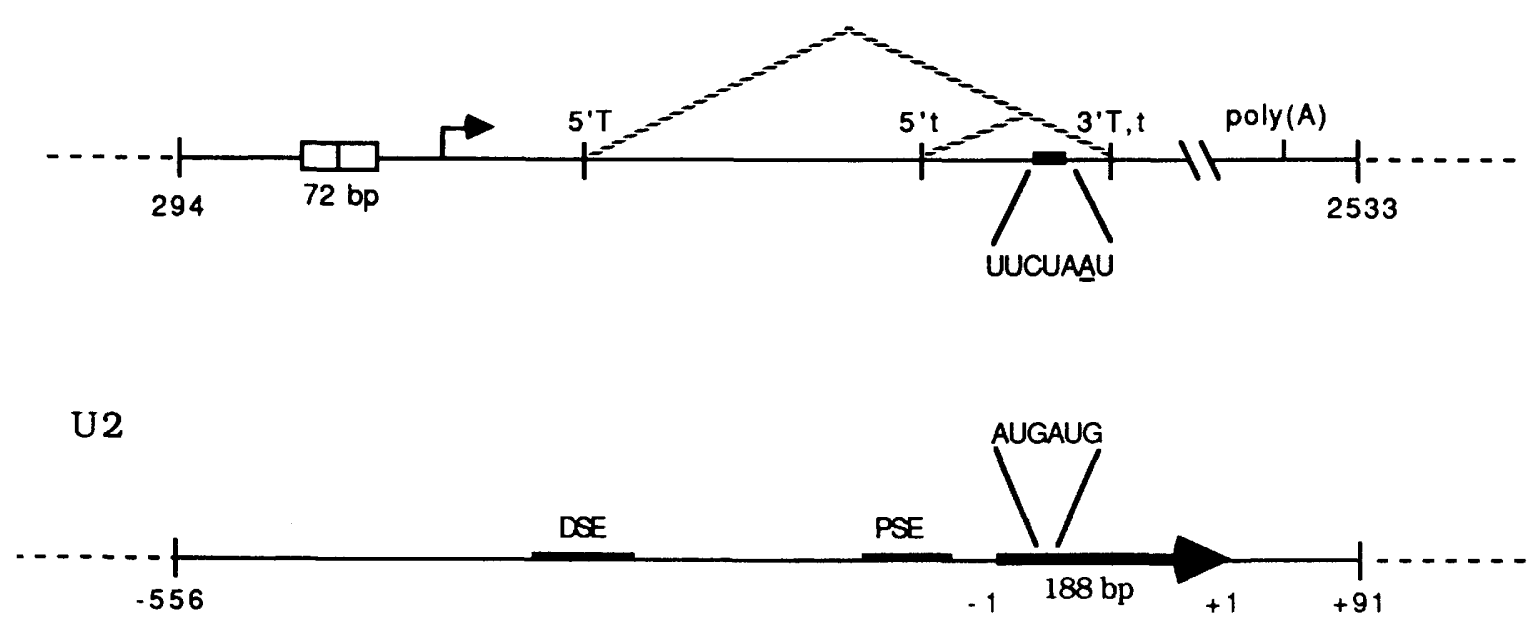

\section{SV40-GGU \\ SV40-AGU \\ SV40-19U \\ SV40-20A/G \\ SV40-21G}

SV40-WT

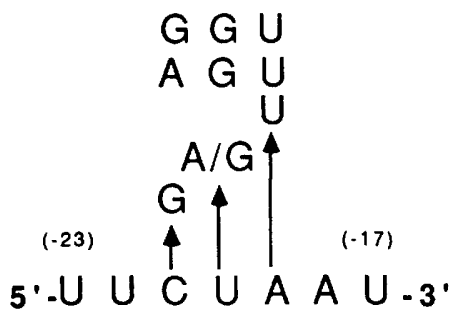

$$
3^{\prime} \cdot \mathrm{A}
$$

(38)

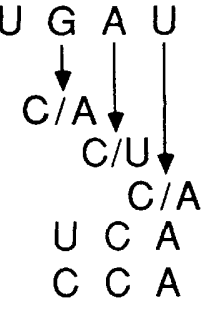

$A \cup C A \quad A$

$A C C A \quad A$
Figure 1. Structure of the SV40 early region and the human U2 snRNA genes, and sequences of the branch site-related mutations. (Top) Structures of the SV40 sequences in the plasmid pSTER are diagramed. Numbers are SV40 coordinates. The promoter/enhancer region is indicated by the 72-bp repeats; $(\rightarrow)$ the transcriptional start site. The large $T$ and small $\mathrm{t} 5^{\prime}$ splice sites, the shared $3^{\prime}$ splice site, and the site of poly(A) addition are indicated. The nucleotide sequence shown below the line is the small $\mathrm{t}$ branch site consensus, with the $\mathrm{A}$ denoting the principal branch acceptor. The $\mathrm{U} 2$ gene is also diagramed. Numbers indicate the number of nucleotides upstream and downstream of the 188-bp U2-coding region. The distal (DSE) and proximal (PSE) promoter elements are indicated. The nucleotide sequence shown above the line is the sequence proposed to base-pair with the branch site consensus. (Bottom) The sequences of the SV40 small $t$ branch site consensus (SV40-WT) and complementary sequences in U2 RNA are indicated. The negative numbers indicate the distance (in nucleotides) upstream of the 3' splice site in the SV40 pre-mRNA; the positive numbers indicate the distance downstream of the U2 RNA 5' end. The base changes introduced in these sequences are listed, and the resulting mutant plasmids were named to indicated these changes. four single-base substitutions were cotransfected with wild-type U2, a potential suppressor, or the non-basepairing $\mathrm{U} 2$ mutant. The results of nuclease $\mathrm{S} 1$ analyses of RNA extracted from these cells is shown in Figure 3. In each case, the efficiency of small $t$ splicing was not significantly affected by cotransfection with wild-type U2, although a small but reproducible increase was observed in the case of one of the two mutations at position -20 (SV40-20G; cf. Figs. 2 and 3). However, all four suppressor U2 snRNAs resulted in significant increases in small $t$ splicing and also substantially increased the ratios of small $t$ to large $T$ mRNA (see Table 1). This latter finding indicates that the effects of the suppressor U2 snRNAs were specific for small $t$ and did not result from a nonspecific enhancement of splicing. Three of the four U2 plasmids containing mutations that do not restore base-pairing potential also enhanced small $t$ splicing, although significantly less well than did the 'suppressor' U2 plasmids (Table 1). In one instance (SV40-20G), this can be explained by the ability of U2-35U to form a G-U base pair, and the lower level of suppression may reflect the relative strengths of G-U versus $\mathrm{G}-\mathrm{C}$ base pairs. The reasons for the increases observed in the other two instances are less clear, although in the case of SV40-20A and U2-35C, the pair that resulted in the largest nonspecific increase, a 'clash' between two opposing adenosines-which might interfere with the overall stability of the presumptive U2-branch site duplex-was eliminated. It may also be that these mutant U2 snRNAs interfere with large $\mathrm{T}$ splicing, resulting in the observed increases in the ratio of small $t$ to large T mRNA.

Because mutations at all of the three positions tested above could be suppressed, we decided to determine whether the triple mutations, which essentially destroy the branch site consensus and totally block small $t$ 


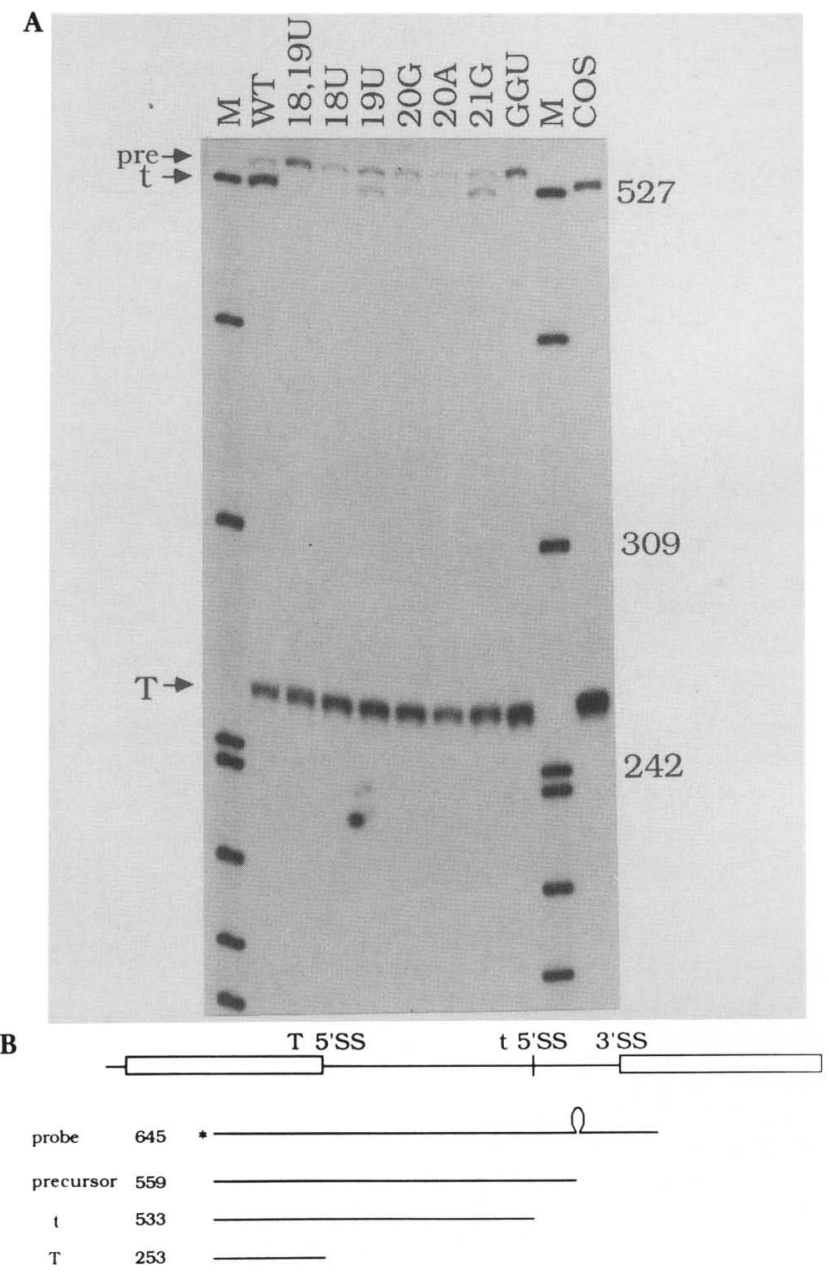

Figure 2. Effects of mutations in the small $\mathrm{t}$ branch site consensus on SV40 early pre-mRNA splicing. (A) Cytoplasmic RNA was extracted from 293 cells transfected with wild-type or indicated branch site mutant plasmids and analyzed by nuclease S1, as described in Materials and methods. (T) Spliced large $\mathrm{T}$ mRNA; $(\mathbf{t})$ small $\mathrm{t}$ mRNA; (pre) unspliced precursor. COS displays an analysis of cytoplasmic RNA extracted from SV40-transformed COS-7 monkey cells. (M) DNA markers (sizes indicated in nucleotides). (B) Diagram of the 3 '-end-labeled DNA probe, which contains an 11-nucleotide insertion between the small $t 5^{\prime}$ splice site and branch site and the protected fragments generated by small $t$, large $T$, and unspliced pre-mRNAs.

splicing, could also be rescued by introduction of the appropriate mutations into U2 snRNA. Therefore, we analyzed two related branch site triple mutations: SV40AGU and SV40-GGU. The A/G transition that distinguishes these two mutants from each other was designed to allow us to investigate further the influence of base-pairing 'strength' on the U2-branch site interaction. Appropriate compensatory mutations were introduced into the $\mathrm{U} 2$ gene, and in addition, quintuple mutations that extend the base-pairing potential of both potential suppressors were constructed also. The sequence changes are all shown in Figure 1, and the results of nu- clease S1 analysis of RNAs from cotransfected cells are shown in Figure 4.

Both of the triple mutants produced virtually no small $t$ mRNA when transfected with wild-type U2. However, cotransfection with the appropriate suppressor plasmids resulted in significant levels of small $\mathbf{t}$ splicing. In fact, the ratio of small $t$ to large T mRNA in the case of SVAGU and U2-UCA was similar to that observed with pSTER (SV40-WT; see Table 1). Interestingly, significant small $\mathbf{t}$ splicing was detected when SV-AGU was cotransfected with U2-CCA, which leaves position -21 in the branch site unpaired. Although the ratio of small $t$ to large $\mathrm{T}$ was lower than observed when all 3 bases could be paired, it was higher than that detected with the unsuppressed mutant $21 G$, which also contains a single mismatch at this position (see Table 1). These results may indicate that a strong C-G base pair at the critical central position of the branch site consensus facilitates use of that site.

The quintuple U2 mutant (U2-AUCAA) also restored small $t$ splicing of SV-AGU. However, the ratio of small $t$ to large $T$ was lower than that observed with U2-UCA. Although we have not excluded the possibility that these mutations alter the stability or structure of the

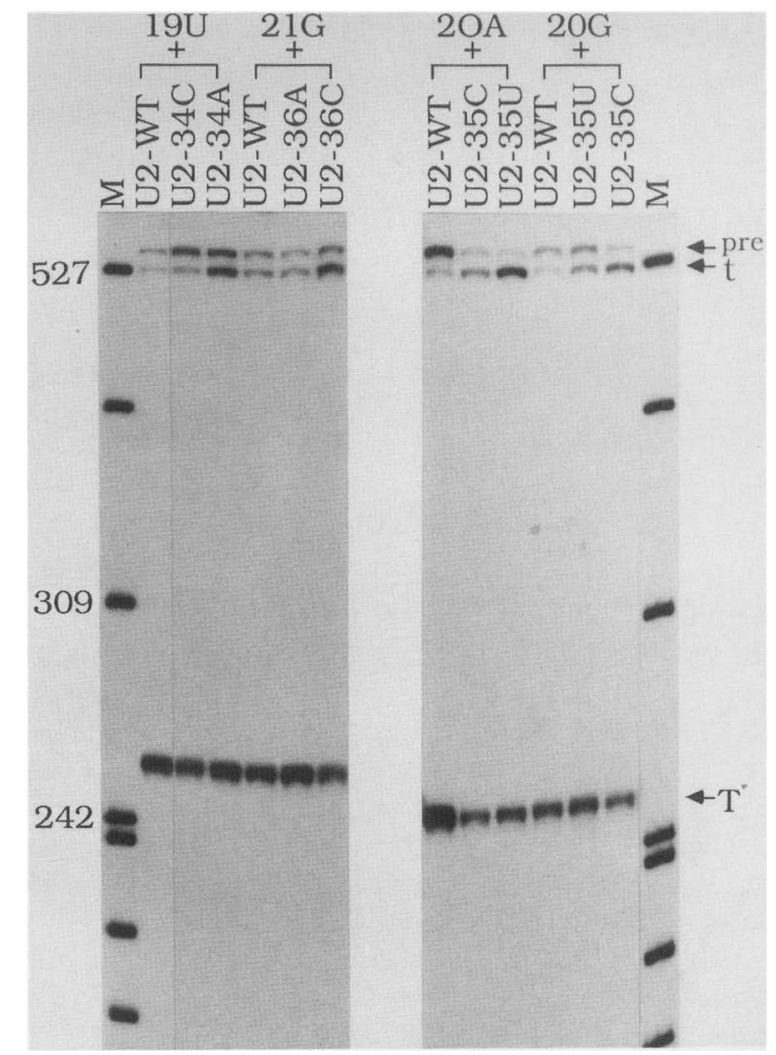

Figure 3. Cotransfection of SV40 single-base substitutions with U2 plasmids. Cytoplasmic RNA extracted from 293 cells transfected with the indicated SV40 mutants plus wild-type or mutated U2 plasmids was analyzed by nuclease S1, as in Fig. 2 . (T) Spliced large T mRNA; ( $t$ ) small t mRNA; (pre) unspliced precursor. 
Table 1. Potential base pairing between wild-type and mutant U2 snRNAs and small $t$ branch sites and the corresponding ratios of small $t$ to large $T$ mRNA

\begin{tabular}{|c|c|c|}
\hline snRNA & Branch site & $\mathrm{t} / \mathrm{T}$ ratio \\
\hline SV40-WT & U U C U A A U & \\
\hline $\mathrm{U} 2-\mathrm{WT}$ & $A \cup G A \cup \quad G$ & 0.90 \\
\hline SV40-19U & U U C U u A U & \\
\hline U2-34A & A U G A a $\quad$ G & 0.49 \\
\hline $\mathrm{U} 2-34 \mathrm{C}$ & $\mathbf{c}$ & 0.14 \\
\hline U2-WT & $\mathrm{U}$ & 0.07 \\
\hline SV40-20A & U U C a A A U & \\
\hline $\mathrm{U} 2-35 \mathrm{U}$ & $A \cup G \mathbf{u} U \quad G$ & 0.86 \\
\hline $\mathrm{U} 2-35 \mathrm{C}$ & c & 0.33 \\
\hline U2-WT & A & 0.03 \\
\hline SV40-20G & U U C g A A U & \\
\hline U2-35C & $A \cup G \mathbf{c} U \quad G$ & 0.53 \\
\hline U2-35U & $\mathbf{u}$ & 0.15 \\
\hline $\mathrm{U} 2-\mathrm{WT}$ & A & 0.09 \\
\hline SV40-21G & UU $g$ U A A U & \\
\hline $\mathrm{U} 2-36 \mathrm{C}$ & $A \cup c A U$ G & 0.52 \\
\hline $\mathrm{U} 2-36 \mathrm{~A}$ & $\mathbf{a}$ & 0.13 \\
\hline $\mathrm{U} 2-\mathrm{WT}$ & G & 0.20 \\
\hline SV40-AGU & $\mathrm{UU}$ a $\mathbf{g} \mathbf{u} \mathrm{AU}$ & \\
\hline U2-UCA & $A \cup \mathbf{u} \mathbf{c} \quad \mathrm{G}$ & 0.84 \\
\hline U2-AUCAA & $\mathbf{a} \mathbf{u} \mathbf{c} \mathbf{a}$ & 0.38 \\
\hline U2-CCA & c c a & 0.31 \\
\hline U2-WT & UGA U G & 0 \\
\hline SV40-GGU & $\mathrm{UU} \mathbf{g} \mathbf{g} \mathbf{u} \mathrm{AU}$ & \\
\hline $\mathrm{U} 2-\mathrm{CCA}$ & $A \cup c c$ a $G$ & 0.09 \\
\hline U2-ACCAA & a c c a a & 0.04 \\
\hline U2-UCA & u c a & 0.12 \\
\hline U2-WT & UGA U G & 0 \\
\hline
\end{tabular}

The SV40 small t pre-mRNA branch site and the complementary region in U2 snRNA are indicated. Lowercase letters indicate the mutated nucleotides. Quantitation of the ratios of small $t$ to large $T(t / T$ ratio) for each pair was determined as described in Materials and methods.

mutant U2 snRNA, one explanation for this is that the additional increase in the base-pairing strength brought about by the extended complementary region of the quintuple mutant actually reduced the efficiency of small $t$ splicing, perhaps by interfering with a subsequent step in the splicing reaction (e.g., dissociation of U2 snRNP). This view is consistent with results obtained with the triple mutant SV-GGU. Although this mutation was clearly suppressed by its cognate suppressor (U2-CCA), the ratio of small $t$ to large $T$ was considerably less than that observed with the related pair, SV-AGU and U2-UCA, and indeed was not significantly different than that detected when SV-GGU was transfected with U2-UCA, which results in a G-U pair instead of a G-C pair (see Fig. 4; Table 1). One explanation for this is that the second C-G base pair reduced small $t$ splicing efficiency, relative to the mutant containing an A-U pair at this position, because the base-pairing interaction was too strong. The corresponding quintuple mutant (U2-ACCAA) again resulted in slightly less efficient small $t$ splicing than did the related triple mutant (U2CCA).

The above results suggest that when a C-G base pair can be formed involving the critical central position in the branch point consensus, the ability to tolerate a mismatch at an adjacent position is increased. To determine whether small $t$ splicing can occur when mismatches are present on both sides of a central C-G base pair, the triple mutant SV-AGU was cotransfected with U2-35C, or, as controls, with several of the other U2 single-base change mutants. The results (shown in Fig. 5) reveal that although a low level of small $t$ splicing was detected in the presence of U2-35C, the efficiency was very low, suggesting that a single, strong base pair cannot effectively compensate for the presence of 2 bases unable to pair. The results shown in Figure 5 also suggest that the suppression we detected is allele-specific, as U2 mutants that were inactive in this experiment were active when cotransfected with the complementary pSTER mutant (see Fig. 3). Further evidence for the specificity of the

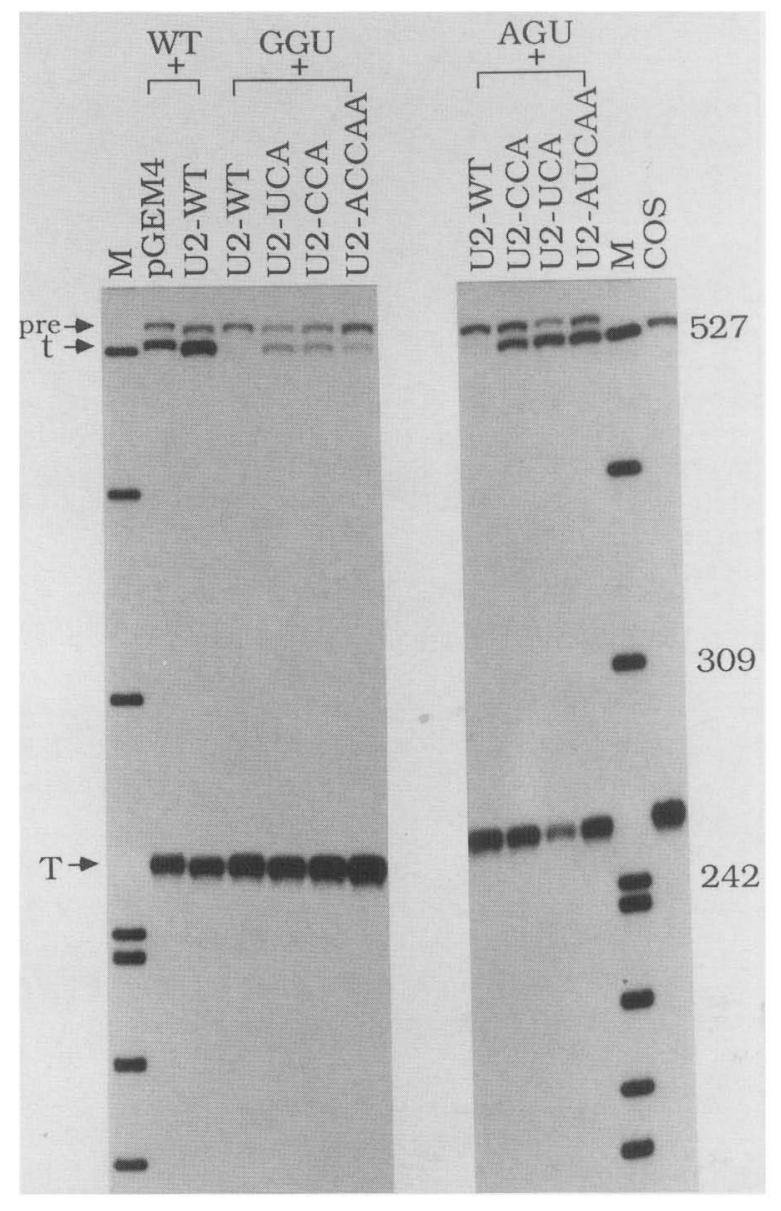

Figure 4. Cotransfection of SV40 triple mutants with wildtype and mutated U2 plasmids. Nuclease S1 analysis of cytoplasmic RNA extracted from 293 cells cotransfected with wildtype SV40 (WT) or the indicated triple base substitution mutants (AGU or GGU) plus the indicated U2 plasmid was performed as in Fig. 2. (T) spliced large T mRNA; $(t)$ small $t$ mRNA; (pre) unspliced pre-mRNAs. 


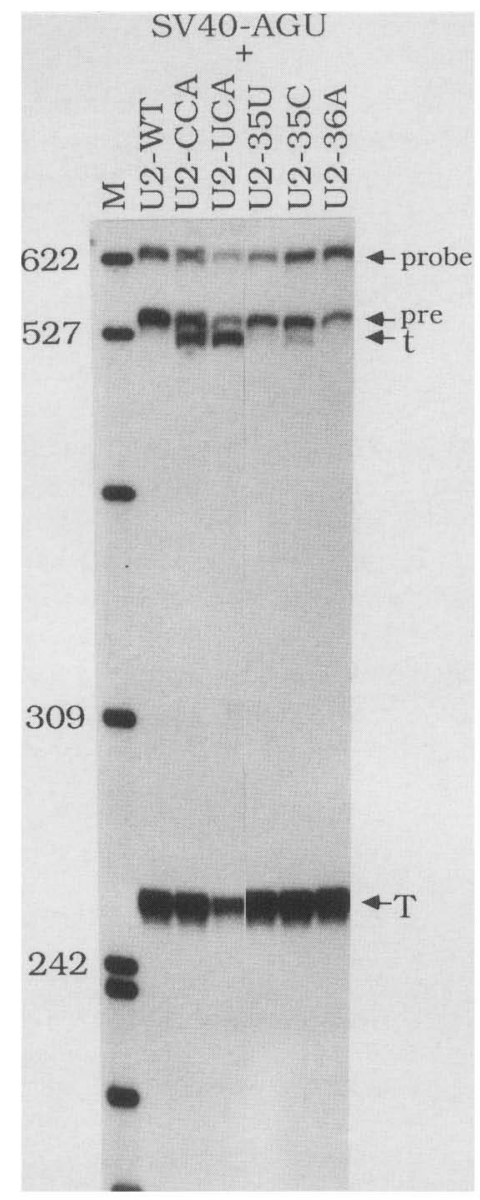

Figure 5. Cotransfection of U2 single-base substitution mutants with the SV40-AGU branch site triple mutant. Nuclease S1 analysis of cytoplasmic RNA extracted from 293 cells cotransfected with SV40-AGU plus the indicated U2 mutant was carried out exactly as in Fig. 2.

U2-branch site interactions described was provided by an experiment in which PSTER was cotransfected with each of the U2 plasmids that were active in the suppression assay. The results, shown in Figure 6, revealed that none of the U2 plasmids increased the ratio of small $t$ to large $\mathrm{T}$ mRNA. Indeed, some of the mutants caused slight decreases in this ratio. This may reflect the ability of some of the suppressors to function preferentially with one or more of the three overlapping, upstream consensus branch site sequences, which can be used only in large T splicing (Noble et al. 1988, 1989).

\section{Discussion}

Our results established that recognition of the mammalian pre-mRNA branch site by U2 snRNP involves basepairing. We presented evidence that the 3 nucleotides immediately $5^{\prime}$ to the branch acceptor pair with nucleotides located 34, 35, and 36 bases from the U2 snRNA $5^{\prime}$ end. Thus, in mammalian cells, as in yeast, pre-mRNA splicing involves base-pairing interactions at both the lariat branch site and the $5^{\prime}$ splice site. How this base- pairing contributes mechanistically to splicing remains to be determined; however, it is interesting that basepairing interactions involving the $5^{\prime}$ splice site and branch site region are also important in group II RNA self-splicing (Schmelzer and Schweyen 1986; Jacquier and Michel 1987). An obvious possibility suggested by previous studies (Schmelzer and Schweyen 1986; Parker et al. 1987; Van der Veen et al. 1987) for the role of basepairing at the branch site is to 'bulge' the branch acceptor from an RNA-RNA helix, which may increase its ability to function as a nucleophile in the trans-esterfication reaction that leads to $5^{\prime}$ splice site cleavage and lariat formation (Cech 1986). Indeed, it has been shown that a single-base insertion in the group II self-splicing bl 1 RNA that causes the branch acceptor A to become base-paired greatly reduces the rate of lariat formation 9 and splicing (Schmelzer and Muller 1987). We suggest that a similar insertion in U2 snRNA would have deleterious effects on nuclear pre-mRNA splicing.

A question raised by our results is whether base pairing between $U 2$ snRNA and the branch site region is necessary and/or sufficient for U2 snRNP binding to the pre-RNA. It is very unlikely that U2 snRNP binding re-

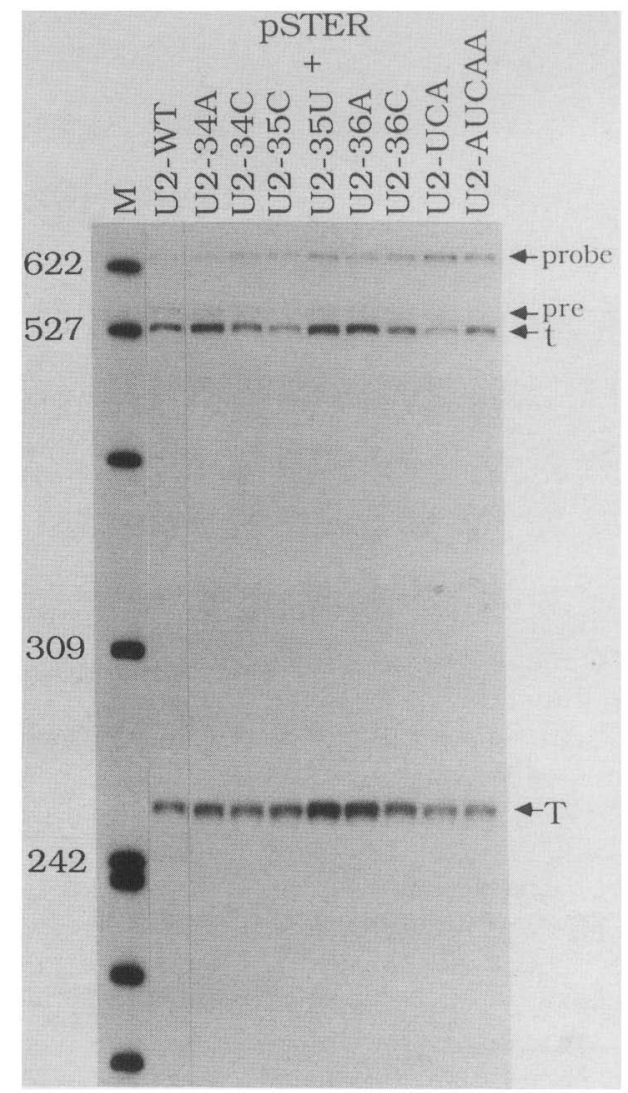

Figure 6. Effects of wild-type and mutant U2 plasmids on splicing of SV40 wild-type pre-mRNA. Nuclease S1 analysis of cytoplasmic RNA extracted from 293 cells cotransfected with SV40 wild-type (pSTER) plus the indicated U2 plasmids was performed as in Fig. 2. (T) Spliced large T mRNA; $(t)$ small $t$ mRNA; (pre) unspliced precursor; (probe) undigested probe. 
quires only this base-pairing interaction. Sequences at the 3 ' splice site consensus are required for U2 snRNP binding to the branch site (Ruskin and Green 1985; Chabot and Steitz 1987), and purified U2 snRNP is unable to bind to pre-mRNA (Ruskin et al. 1988). A more interesting question is whether base-pairing is, in all cases, necessary for interaction of $\mathrm{U} 2$ snRNP with the pre-mRNA. The fact that cryptic branch sites need bear little resemblance to the consensus (Padgett et al. 1985; Ruskin and Green 1985) is consistent with the notion that it may not be, although the potential for G-U base pairing may, in most cases, allow formation of several base pairs. Additional studies will be required to determine the role of base pairing in such cases, as well as to investigate the influence of base-pairing 'strength' on branch site selection.

A related question is whether base pairing is important for recognition of the branch site by U2 snRNP, for the function of the particle in splicing, or for both. We believe that the latter is most likely to be correct. As discussed above, the analogy with group II self-splicing introns suggests that the presence of the branch acceptor adenosine in a helix bulge may be important for its function in splicing. The branch site consensus also appears to serve as a recognition signal. For example, the SV40 early pre-RNA branch site region contains four consensus sequences, each of which can interact with U2 snRNP and function as a lariat branch site (Noble et al. 1988, 1989). It is interesting that branch site-U2 snRNA base pairing is apparently not required during the earliest stages of the splicing reaction in vitro, i.e., during spliceosome assembly. An internal region of U2 snRNA, containing the sequence complementary to the branch site, is not required until a step subsequent to U2 snRNP binding to the pre-RNA (Frendeway et al. 1987). Likewise, mutations in the branch site consensus that inhibit splicing do not affect the assembly of spliceosomes (Reed and Maniatis 1988). These results suggest a model in which the initial binding of U2 snRNP to the pre-RNA is through interaction with other factors, most likely bound to the adjacent polypyrimidine stretch (e.g., Kramer 1988; Ruskin et al. 1988). Following this preliminary interaction, we suggest that binding is stabilized by base pairing, which determines the precise site of branch formation. Without this interaction, the first step of splicing does not occur, and, presumably, the spliceosome dissociates.

Does the branch site consensus serve any other function than to base-pair with U2 snRNA? All of the mutations analyzed here could be suppressed, and in all cases but one, the efficiency of small $t$ splicing was restored to near wild-type levels. This is despite the fact that the newly synthesized suppressor U2 snRNPs must compete with endogenous U2 snRNPs, which would be able to interact with upstream branch sites, resulting in large $\mathrm{T}$ splicing. That one mutation (the triple mutant SVGGU) could only be suppressed inefficiently does not argue that the mutated nucleotides play a role in splicing in addition to base-pairing with U2 snRNA, because the related triple mutant (SV-AGU), in which the same 3 nucleotides in the branch site consensus had been mutated, was suppressed very efficiently. We believe that the different suppression efficiencies displayed by these two mutants reflects, instead, important differences in the U2 snRNA-branch site base pairing that influence splicing efficiencies (see below). Thus, we interpret the results presented here as supporting the view that base pairing with U2 snRNA may be the only function of at least the three positions in the consensus that were analyzed. This hypothesis is supported by observations in other systems. One mutation in the group II self-splicing yeast mitochondrial bl 1 intron (Schmelzer and Schweyen 1986), two in the yeast HIS4 gene (Parker et al. 1987), and one in the human $\beta$-globin gene (Zhuang and Weiner 1989), which disrupt branch site base pairing, can all be suppressed by the appropriate compensatory mutations. This contrasts to the situation at the $5^{\prime}$ consensus splice sites of yeast and metazoan nuclear genes, where mutations at certain positions cannot be suppressed by compensatory mutations in U1 RNA (Zhuang and Weiner 1986; Siliciano and Guthrie 1988).

Our results have shown that the selection of alternative $5^{\prime}$ splice sites in a pre-mRNA can be influenced by a specific trans-acting factor (U2 snRNP), acting at the lariat branch site. In similar experiments using mutant human $\beta$-globin pre-mRNAs, Zhuang and Weiner (1989) have shown that the choice of $3^{\prime}$ splice site can be controlled by a similar interaction. These observations raise the possibility that differential interactions between U2 snRNPs and lariat branch sites might naturally influence alternative splicing pathways. The simplest scenario for such regulation might be that different U2 snRNAs exist containing nucleotide changes that would alter base-pairing. However, to date, no U2 RNA sequence variants of any sort have been described (for review, see Mattaj and Hamm 1989|. However, it is possible that other factors might influence the U2 snRNPbranch site interaction. Specifically, we have shown that mutations in the polypyrimidine stretch can influence alternative splicing of SV40 early pre-mRNA (Fu et al. 1988b). Additionally, several factors have been implicated in binding to the polypyrimidine stretch $-3^{\prime}$ splice site (Chabot et al. 1985; Gerke and Steitz 1986; Tazi et al. 1986; Ruskin et al. 1988; Swanson and Dreyfuss 1988). Perhaps interactions at this site might influence $\mathrm{U} 2$ snRNP binding to the branch site in a manner that reflects the strength of the branch site-U2 snRNA base pairing. Indeed, the results described here support the notion that the strength of this base pairing can influence alternative splicing.

In this regard, it is interesting to compare the metazoan branch site $-3^{\prime}$ splice site region with that found in yeast. The UACUAAC sequence in yeast appears to be absolutely conserved, and its integrity is crucial for splicing. However, yeast introns lack a conserved polypyrimidine stretch at their $3^{\prime}$ splice sites, and mutations that destroy the $3^{\prime}$ splice site do not interfere with spliceosome assembly (Rymond et al. 1987) or the first step of splicing (Rymond and Rosbash 1985). An interesting possibility is that these differences reflect the apparent 
lack of alternative splicing pathways in yeast. Perhaps the assembly of alternative spliceosomes, which must occur during alternative splicing, requires flexibility in the U2 snRNP $\rightarrow$ branch site interaction in some way. This may be particularly so in cases of regulated alternative splicing where subtle perturbations might lead to changes in splicing patterns. Future studies should provide more insight into the role of U2 snRNP-branch site interactions in alternative splicing, as well as the function of $\mathrm{U} 2$ snRNA-branch site base-pairing in splicing per se.

\section{Materials and methods}

\section{Plasmid constructions}

SV40 branch site mutants were derived from the plasmid pSTER (Lewis and Manley 1985), which contains the entire SV40 early region from a KpnI site at SV40 nucleotide 294 (Tooze 1981) to a BamHI site at nucleotide 2533, inserted between the EcoRI and BamHI sites of pBR322. The template for oligonucleotide-directed mutagenesis was constructed by subcloning an 1169-nucleotide HindIII fragment of SV40 (nucleotides 5171-4002) into M13mp18. Mutagenesis was performed essentially as described by Kunkel et al. (1987). Mutants were identified by DNA sequencing (Sanger et al. 1977), using a primer located 70 nucleotides upstream of the region mutagenized (Noble et al. 1988). Mutated DNA was recloned into pSTER by ligating BstXI-PfIMI fragments (SV40 nucleotides 4759-4558), together with PflMI-BamHI (SV40 nucleotides $4558-2533$ ) and BamHI-BstXI (pBR322 nucleotide 375 to SV40 nucleotide 4759 ) fragments derived from pSTER.

Human U2 wild-type, kindly provided by E. Lund and J. Dahlberg, and mutants $34 \mathrm{~A}, 34 \mathrm{C}$, and $36 \mathrm{~A}$ were constructed by cloning a HindIII fragment /created by attaching linkers at HincII and NaeI sites, which correspond respectively to positions -258 and +94 relative to the coding region; see Westin et al. 1984) into pGEM4. The template for oligonucleotide-directed mutagenesis was constructed by subcloning the same HindIII fragment into $\mathrm{M} 13 \mathrm{mp} 19$. Mutagenesis was carried out as described above. $\mathrm{U} 2$ mutants $35 \mathrm{U}, 35 \mathrm{C}$, and $36 \mathrm{C}$ in $\mathrm{M} 13 \mathrm{mp} 8$ phage were kindly provided by Y. Zhuang and A.M. Weiner and were cloned into pGEM4 using EcoRI and PstI site (from M13mp8 linker). These plasmids contain 556 bp of U2 5'flanking sequence, the 188-bp coding region, and 91 bp of 3'flanking sequence (Yuo et al. 1985). The remaining U2 mutants, U2-CCA, U2-UCA, U2-ACCAA, and U2-AUCAA, were constructed by inserting the EcoRI-PstI fragment from mutant $36 \mathrm{C}$ into $\mathrm{Ml} 3 \mathrm{mpl} 9$ for site-directed mutagenesis and cloning the resulting mutants into pGEM4.

\section{Transfection and RNA analysis}

The 293 cells were grown in Dulbecco's modified Eagle's medium, supplemented with $10 \%$ fetal bovine serum. Cell transfection was performed as described (Lewis and Manley 1985; Fu and Manley 1987), except that either $25 \mu \mathrm{g}$ pSTER (or derived mutants), or $10 \mu \mathrm{g}$ pSTER (or derived mutants) plus $40 \mu \mathrm{g}$ of the indicated U2 plasmid or pGEM carrier DNA was used. Transient expression was terminated $48 \mathrm{hr}$ after DNA addition, and cytoplasmic RNA was isolated essentially as described by $\mathrm{Fu}$ and Manley (1987). Nuclease S1 analysis also was performed essentially as described by Fu and Manley (1987), using a 3'. end-labeled HindIII-BsmI fragment probe (nucleotides $5171-4528$ ) isolated from plasmid pSTER-il1 (Fu and Manley
1987), which contains an 11 -nucleotide insertion at nucleotide 4612 , between the small $\mathrm{t} 5^{\prime}$ splice site and branch site region. The use of this probe permitted resolution of unspliced premRNA, which was present in some experiments due to nuclear leakage, and undigested probe. Nuclease S1 resistant products were resolved by electrophoresis through $5 \%$ polyacrylamide$7 \mathrm{M}$ urea gels, which were dried and exposed to X-ray film at $-70^{\circ} \mathrm{C}$ in the presence of intensifying screens. Quantitation of small $t$ and large $T$ mRNAs was performed by densitometry scanning using a Bio-Rad model 1650 densitometer equipped with a program to correct for nonlinearities resulting from the use of intensifying screens, kindly provided by L. Chasin.

\section{Acknowledgments}

We are grateful to S. Connelly and $\mathrm{H}$. Ge for their valuable technical advice and helpful discussions and to Y.-M. Han and $\mathrm{H}$. Ge for their contributions during the early phase of this work. We also thank J. Noble, X.-Y. Fu, L. Ryner, K. Han, Y. Takagaki, D. Read, J. Colgan, and J. Harper for their suggestions and advice during the course of this work and $C$. Prives and Z.-Q. Pan for valuable discussions. We are most grateful to $\mathrm{Y}$. Zhuang and A.M. Weiner for providing $\mathrm{U} 2$ plasmids 36C, 35C, and $35 \mathrm{U}$, for many useful discussions, and for sharing their results with us prior to publication. We also thank E. Lund and J. Dahlberg for providing the wild-type U2 plasmid. We thank L. Chasin for the use of his densitometer, M. Kopczynski, L. Lee, and $M$. Wong for excellent technical assistance, and T. Coudreaut for preparing the manuscript. This work was supported by grant CA-33620 from the National Institutes of Health.

\section{References}

Aebi, M., H. Homig, and C. Weissmann. 1987. 5' cleavage site in eukaryotic pre-mRNA splicing is determined by the overall $5^{\prime}$ splice region, not by the conserved 5' GU. Cell 50: $237-246$.

Bindereif, A. and M.R. Green. 1987. An ordered pathway of snRNP binding during mammalian splicing complex assembly. EMBO I. 6: 2514-2524.

Black, D.L., B. Chabot, and J.A. Steitz. 1985. U2 as well as U1 small nuclear ribonucleoproteins are involved in pre-mRNA splicing. Cell 42: 737-750.

Cech, T.R. 1986. The generality of self-splicing RNA: Relationship to nuclear mRNA splicing. Cell 44: 207-210.

Chabot, B. and J.A. Steitz. 1987. Multiple interactions between the splicing substrate and small nuclear ribonucleoproteins in spliceosomes. Mol. Cell. Biol. 7: 281-293.

Chabot, B., D.L. Black, D.M. LeMaster, and J.A. Steitz. 1985. The $3^{\prime}$ splice site of pre-messenger RNA is recognized by a small nuclear ribonucleoprotein. Science 230: 1344-1349.

Frendewey, D., A. Kramer, and W. Keller. 1987. Different small ribonucleoprotein particles are involved in different steps of splicing complex formation. Cold Spring Harbor Symp. Quant. Biol. 52: 287-298.

Fu, X.Y. and J.L. Manley. 1987. Factors influencing alternative splice site utilization in vivo. Mol. Cell. Biol. 7: 738-748.

Fu, X.-Y., J. Colgan, and J.L. Manley. 1988a. Multiple cis-acting sequence elements are required for efficient splicing of SV40 small t pre-mRNA. Mol. Cell. Biol. 8: 3582-3590.

Fu, X.-Y., H. Ge, and J.L. Manley. 1988b. The role of the polypyrimidine stretch at the SV40 early pre-mRNA 3' splice site in alternative splicing. EMBO J. 7: 809-817.

Gerke, V. and J.A. Steitz. 1986. A protein associated with small nuclear ribonucleoprotein particles recognizes the $3^{\prime}$ splice site of pre-messenger RNA. Cell 47: 973-984. 
Graham, F.L., J. Smiley, W.C. Russell, and R. Nairn. 1977. Characteristics of a human cell line transformed by DNA from human adenovirus type 5. J. Gen. Virol. 36: 59-72.

Green, M.R. 1986. Pre-mRNA splicing. Annu. Rev. Genet. 20: $671-708$.

Jacquier, A. and F. Michel. 1987. Multiple exon-binding sites in class II self-splicing introns. Cell 50: 17-29.

Keller, E.B. and W.A. Noon. 1984. Intron splicing: A conserved internal signal in introns of animal pre-mRNAs. Proc. Natl. Acad. Sci. 81: 7417-7420.

Kramer, A. 1988. Presplicing complex formation requires two proteins and U2 snRNP. Genes Dev. 2: 1155-1167.

Kunkel, T.A., J.D. Roberts, and R.A. Kakour. 1987. Rapid and efficient site-specific mutagenesis without phenotypic selection. Methods Enzymol. 154: 367-382.

Langford, C.J. and D. Gallwitz. 1983. Evidence for an introncontained sequence required for the splicing of yeast RNA polymerase II transcripts. Cell 33: 519-527.

Lerner, M.R., J.A. Boyle, S.M. Mount, S.L. Wolin, and J.A. Steitz. 1980. Are snRNPs involved in splicing? Nature 283: 220-224.

Lewis, D. and J.L. Manley. 1985. Repression of simian virus 40 early transcription by viral DNA replication in human 293 cells. Nature 317: 172-175.

Maniatis, T. and R. Reed. 1987. The role of small nuclear ribonucleoprotein particles in pre-mRNA splicing. Nature 325: $673-678$.

Mattaj, I.W. and J. Hamm. 1989. Regulated splicing in early development and stage-specific U snRNPs. Development 105: $183-189$.

Mount, S.M., I. Petterson, M. Hinterberger, A. Karmas, and J.A. Steitz. 1983. The U1 small nuclear RNA-protein complex selectively binds a $5^{\prime}$ splice site in vitro. Cell 33: 509-518.

Noble, J.C.S., H. Ge, M. Chaudhuri, and J.L. Manley. 1989. Factor interactions with the SV40 early pre-mRNA influence branch point selection and alternative splicing. Mol. Cell. Biol. 9: 2007-2017.

Noble, J.C.S., Z.-Q. Pan, C. Prives, and J.L. Manley. 1987. Splicing of SV40 early pre-mRNA to large $\mathrm{T}$ and small $\mathrm{t}$ mRNAs utilizes different patterns of lariat branch sites. Cell 50: $227-236$.

Noble, J.C.S., C. Prives, and J.L. Manley. 1988. Alternative splicing of SV40 early pre-mRNA is determined by branch site selection. Genes Dev. 2: 1460-1475.

Padgett, R.A., P.J. Grabowski, M.M. Konarska, S. Seiler, and P.A. Sharp. 1986. Splicing of messenger RNA precursors. Annu. Rev. Biochem 55: 1119-1150.

Padgett, R.A., M.M. Konarska, M. Aebi, H. Hornig, C. Weissmann, and P.A. Sharp. 1985. Nonconsensus branch-site sequences in the in vitro splicing of transcripts of mutant rabbit $\beta$-globin genes. Proc. Natl. Acad. Sci. 82: 8349-8353.

Parker, R., P.G. Siliciano, and C. Guthrie. 1987. Recognition of the TACTAAC box during mRNA splicing in yeast involves base pairing of the U2-like snRNA. Cell 49: 229-239.

Pikielny, C.W., J.L. Teem, and M. Rosbash. 1983. Evidence for the biochemical role of an internal sequence in yeast nuclear mRNA introns: Implications for U1 RNA and metazoan mRNA splicing. Cell 34: 395-403.

Reed, R. and T. Maniatis. 1988. The role of the mammalian branch point sequence in pre-mRNA splicing. Genes Dev. 2: $1268-1276$.

Rogers, J. and R. Wall. 1980. A mechanism for RNA splicing. Proc. Natl. Acad. Sci. 71: 1877-1879.

Ruskin, B. and M.R. Green. 1985. Role of the 3' splice site consensus sequence in mammalian pre-mRNA splicing. Nature 317: $732-734$.
Ruskin, B., J.M. Green, and M.R. Green. 1985. Cryptic branch point activation allows accurate in vitro splicing of human $\beta$-globin intron mutants. Cell 41: 833-844.

Ruskin, B., P.D. Zamore, and M.R. Green. 1988. A factor, $\mathrm{U} 2 \mathrm{AF}$, is required for $\mathrm{U} 2 \mathrm{snRNP}$ binding and splicing complex assembly. Cell 52: 207-219.

Ruskin, B., A.R. Krainer, T. Maniatis, and M.R. Green. 1984. Excision of an intact intron as a novel lariat structure during pre-mRNA splicing in vitro. Cell 38: 317-331.

Rymond, B.C. and M. Rosbash. 1985. Cleavage of 5' splice site and lariat formation are independent of $3^{\prime}$ splice site in yeast mRNA splicing. Nature 317: 735-737.

Rymond, B.C., D.D. Torrey, and M. Rosbash. 1987. A novel role for the $3^{\prime}$ region of introns in pre-mRNA splicing of Saccharomyces cerevisiae. Genes Dev. 1: 238-246.

Sanger, F., S. Nicklen, and A.R. Coulson. 1977. DNA sequencing with chain-terminating inhibitors. Proc. Natl. Acad. Sci. 74: 5463-5467.

Schmelzer, C. and M.W. Muller. 1987. Self-splicing of group II introns in vitro: Lariat formation and 3' splice site selection in mutant RNAs. Cell 51: 753-762.

Schmelzer, C. and R.J. Schweyen. 1986. Self-splicing of group II introns in vitro: Mapping of the branch point and mutational inhibition of lariat formation. Cell 46: 557-565.

Seraphin, B., L. Kretzner, and M. Rosbash. 1988. A U1 snRNA : pre-mRNA base pairing interaction is required early in yeast spliceosome assembly but does not uniquely define the 5' cleavage site. EMBO $1.7: 2533-2538$.

Siliciano, P.G. and C. Guthrie. 1988. 5' splice site selection in yeast: Genetic alternations in base-pairing with $\mathrm{U} 1$ reveal additional requirements. Genes Dev. 2: 1258-1267.

Swanson, M.S. and D. Dreyfuss. 1988. RNA binding of hnRNP proteins: A subset bind to the $3^{\prime}$ end of introns. EMBO $\mathrm{J}$. 7: 3519-3529.

Tazi, J., C. Alibert, J. Temsamani, I. Reveilland, G. Cathala, C. Burnel, and P. Jeanteur. 1986. A protein that specifically recognizes the 3 ' splice site of mammalian pre-mRNA introns is associated with a small nuclear ribonucleoprotein. Cell 47: $755-766$.

Tooze, J. 1981. DNA tumor viruses. 2nd ed. Cold Spring Harbor Laboratory, Cold Spring Harbor, New York.

Van der Veen, R., J.H.J.M. Kwakman, and L.A. Grivell. 1987. Mutations at the lariat acceptor site allow self-splicing of group II intron without lariat formation. EMBO $/$. 6: 38273831.

Weber S. and M. Aebi. 1988. In vitro splicing of mRNA precursor: $5^{\prime}$ cleavage site can be predicted from the interaction between the $5^{\prime}$-terminus of U1 snRNA. Nucleic Acids Res. 16: 471-486.

Westin, G., E. Lund, J.T. Murphy, U. Pettersson, and J.E. Dahlberg. 1984. Human U2 and U1 RNA genes use similar transcription signals. EMBO I. 3: 3295-3301.

Wieringa, B., E. Hofer, and C. Weissmann. 1984. A minimal intron length but no specific internal sequence is required for splicing the large rabbit $\beta$-globin intron. Cell 37: 915-925.

Yuo, C.-Y., M. Ares, Jr., and A.M. Weiner. 1985. Sequences required for $3^{\prime}$ end formation of human U2 small nuclear RNA. Cell 42: 193-202.

Zeitlin, S. and A. Efstratiadis. 1984. In vitro splicing products of the rabbit $\beta$-globin pre-mRNA. Cell 39: 589-602.

Zhuang, Y. and A.M. Weiner. 1986. A compensatory base change in U1 snRNA suppresses a $5^{\prime}$ splice site mutation. Cell 46:827-835.

Zhuang, Y., A.M. Goldstein, and A.M. Weiner. 1989. UACUAAC is the preferred branch site for mammalian mRNA splicing. Proc. Natl. Acad. Sci. 86: 2752-2756. 


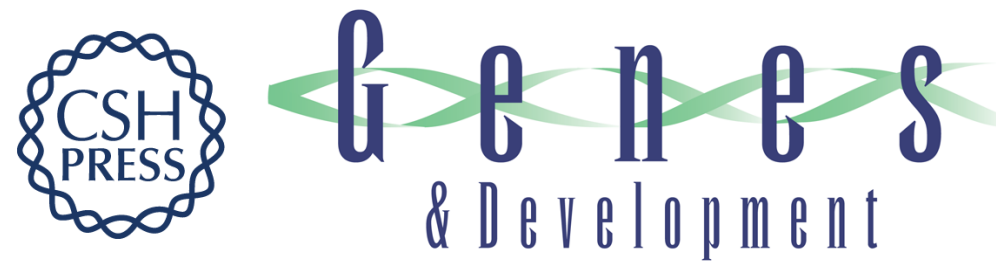

\section{Mammalian pre-mRNA branch site selection by U2 snRNP involves base pairing.}

$\mathrm{J} \mathrm{Wu}$ and $\mathrm{J} L$ Manley

Genes Dev. 1989, 3:

Access the most recent version at doi:10.1101/gad.3.10.1553

References This article cites 53 articles, 15 of which can be accessed free at:

http://genesdev.cshlp.org/content/3/10/1553.full.html\#ref-list-1

License

Email Alerting

Service

Receive free email alerts when new articles cite this article - sign up in the box at the top right corner of the article or click here.

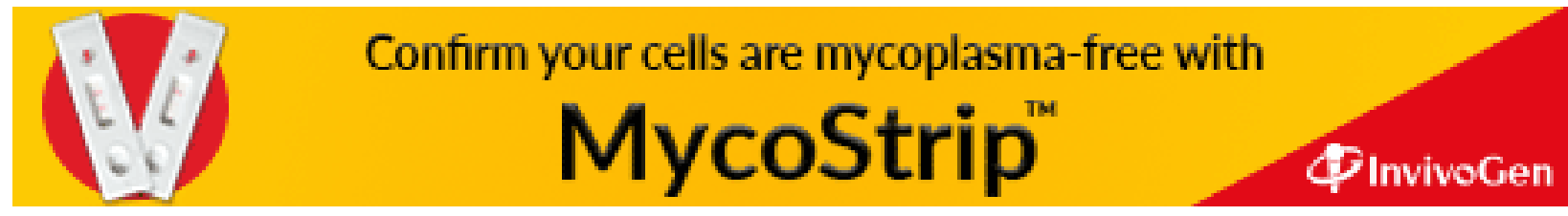

